\title{
The small GTPase RhoA, but not Rac1, is essential for conditioned aversive memory formation through regulation of actin rearrangements in rat dorsal hippocampus
}

\author{
Jun WANG ${ }^{1}$, Yu-hua WANG ${ }^{1,3}$, Yuan-yuan $\mathrm{HOU}^{2}$, Tao XI ${ }^{1, *}$, Yao $\mathrm{LIU}^{2, *}$, Jing-gen LIU ${ }^{2}$ \\ ${ }^{1}$ Biotechnology Center, School of Life Science and Technology, China Pharmaceutical University, Nanjing 210009, China; ${ }^{2}$ State Key \\ Laboratory of Drug Research, Shanghai Institute of Materia Medica, Shanghai Institute for Biological Sciences, Chinese Academy of \\ Sciences, Shanghai 201203, China; ${ }^{3}$ School of Pharmacy, Nanjing University of Chinese Medicine, Nanjing 210046, China
}

\begin{abstract}
Aim: Actin rearrangements are induced in the dorsal hippocampus after conditioned morphine withdrawal, and involved in the formation of conditioned place aversion. In the present study, we investigated the mechanisms underlying the actin rearrangements in rat dorsal hippocampus induced by conditioned morphine withdrawal.

Methods: The RhoA-ROCK pathway inhibitor Y27632 (8.56 $\mu \mathrm{g} / 1 \mu \mathrm{L}$ per side) or the Rac1 inhibitor NSC23766 (25 $\mu \mathrm{g} / 1 \mu \mathrm{L}$ per side) was microinjected into the dorsal hippocampus of rats. Conditioned place aversion (CPA) induced by naloxone-precipitated morphine withdrawal was assessed. Crude synaptosomal fraction of hippocampus was prepared, and the amount of F-actin and G-actin was measured with an Actin Polymerization Assay Kit.

Results: Conditioned morphine withdrawal significantly increased actin polymerization in the dorsal hippocampus at $1 \mathrm{~h}$ following the naloxone injection. Preconditioning with microinjection of Y27632, but not NSC23766, attenuated CPA, and blocked the increase in actin polymerization in the dorsal hippocampus.

Conclusion: Our results suggest that the small GTPase RhoA, but not Rac1, in the dorsal hippocampus is responsible for CPA formation, mainly through its regulation of actin rearrangements.
\end{abstract}

Keywords: morphine withdrawal; aversive memory; conditioned place aversion; dorsal hippocampus; actin rearrangement; RhoA; Rac1; naloxone; Y27632; NSC23766

Acta Pharmacologica Sinica (2013) 34: 811-818; doi: 10.1038/aps.2013.3; published online 8 Apr 2013

\section{Introduction}

Opiate addiction is a chronic relapsing disorder characterized by compulsive drug seeking and taking behaviors ${ }^{[1]}$, which are driven by two distinct motivational factors: the rewarding effects of the drug (positive reinforcement) and the avoidance of withdrawal symptoms (negative reinforcement). Clinical evidence suggests that the negative reinforcement associated with drug withdrawal may play a more important role in driving persistent drug craving and relapse to compulsive drug use $^{[2,3]}$. Conditioned place aversion (CPA) is a model of Pavlovian associative learning in which drug withdrawal is paired with a particular context that then triggers the onset of asso-

\footnotetext{
* To whom correspondence should be addressed.

E-mail xi-tao18@hotmail.com (Tao XI);

liuyaowuhan@163.com (Yao LIU)

Received 2012-10-23 Accepted 2013-01-11
}

ciation between the negative effects of drug withdrawal with the environment, leading the animals to avoid the withdrawalpaired context. Therefore, CPA is one of the most sensitive animal models for measurement of the negative effects of morphine withdrawal in dependent animals ${ }^{[4]}$ and can be used to explore the neurobiological mechanisms underlying aversive memory formation of drug withdrawal.

Multiple lines of evidence have demonstrated that the hippocampus plays an important role in learning and memory in both animals and humans ${ }^{[5-7]}$, and the hippocampus may be a major target for studies of synaptic plasticity and information storage in the brain. We recently found that excitotoxic lesions of the dorsal hippocampus impaired morphine withdrawal-induced CPA, confirming the crucial role of the hippocampus in the motivational effects of opioid withdrawalassociated memory ${ }^{[8]}$. This hippocampal synaptic plasticity contributes to a variety of brain functions including learning 
and memory ${ }^{[9,10]}$. Changes in dendritic spine morphology are believed to be involved in synaptic plasticity ${ }^{[11,12]}$. Cytoskeletal actin is the major structural component of the dendritic spine ${ }^{[13]}$, and its dynamic conversion between G-actin (globular actin) and F-actin (actin filament), namely actin rearrangements, is involved in synaptic plasticity ${ }^{[14,15]}$. A growing body of evidence suggests that actin rearrangements play a crucial role in synaptic plasticity associated with learning and memory ${ }^{[16,17]}$. Our previous work also showed in the rat CPA model that synaptic actin rearrangements in the dorsal hippocampus were required for aversive memory formation ${ }^{[8]}$. However, the molecular mechanisms of actin rearrangements in the dorsal hippocampus, which are critical for aversive memory formation, have yet to be elucidated.

Rho, Rac, and Cdc42 are three members of the small Rho GTPase family. They oscillate between a GTP-bound active state and a GDP-bound inactive state and act as molecular switches that control the organization and dynamics of the actin cytoskeleton ${ }^{[18]}$. Much evidence has demonstrated that the Rho GTPases control the assembly and disassembly of the actin cytoskeleton and regulate actin rearrangements at synapses in response to extracellular signals ${ }^{[19,20]}$. These findings suggest the possibility that the Rho GTPases may be involved in synaptic actin polymerization. Therefore, in the present study, we utilized the CPA model to explore whether and which Rho GTPases are responsible for the actin rearrangements in the dorsal hippocampus, as well as aversive memory formation of drug withdrawal.

\section{Materials and methods}

Animals

Sprague-Dawley male rats (clean grade) weighing 220-300 g were obtained from the Laboratory Animal Center, Chinese Academy of Sciences (Shanghai, China). Rats were housed two to three per cage and maintained on a $12 \mathrm{~h}$ light/dark cycle with access to food and water ad libitum. All experimental procedures were in strict accordance with the National Institutes of Health Guide for the Care and Use of Laboratory Animals.

\section{Conditioned place aversion}

The CPA apparatus (Anilab Software \& Instruments Co, Ltd, China) was divided into two equal-sized compartments [55 $\mathrm{cm}$ (length) $\times 30 \mathrm{~cm}$ (width) $\times 30 \mathrm{~cm}$ (height)] separated by a removable board $(10 \times 10 \mathrm{~cm})$, which allowed the rats free access to each compartment. The two compartments were distinguished by visual and tactile cues: one was a blank wall with a smooth floor, whereas the other was a white wall with a textured floor. These distinctive tactile and visual stimuli served as the conditioning cues. The conditioned morphine withdrawal procedure has been described previously ${ }^{[8,21]}$. Briefly, animals experienced three phases: preconditioning, conditioning, and testing. In the preconditioning phase, the rats were allowed to freely explore the entire apparatus for $15 \mathrm{~min}$. Time spent in each compartment was recorded, and those showing a strong unconditioned aversion (one compartment $>720 \mathrm{~s}$ ) for either compartment were eliminated from the study. Conditioning took place over the next $2 \mathrm{~d}$. On the first day, the rats were injected with saline $(1 \mathrm{~mL} / \mathrm{kg}, \mathrm{sc})$ and then returned to home cages. Four hours later, they were given saline again and then confined to either compartment in a counterbalanced manner for $30 \mathrm{~min}$. On the second day, the rats were injected with either morphine $(10 \mathrm{mg} / \mathrm{kg}$, sc) or saline $(1 \mathrm{~mL} / \mathrm{kg}, \mathrm{sc})$ and then returned to their home cages. Four hours later, they were injected with either naloxone $(0.3$ $\mathrm{mg} / \mathrm{kg}$, sc) or saline and then confined to the compartment opposite to the first day for $30 \mathrm{~min}$. This compartment will be referred to as the "drug treatment-paired compartment." The testing phase took place $24 \mathrm{~h}$ after the conditioning trial. During this phase, all of the rats were allowed to freely explore the entire apparatus for $15 \mathrm{~min}$, and the amount of time spent in each compartment was recorded. The CPA score represents the time in the drug treatment-paired compartment during the testing phase minus that during the preconditioning phase. For Western blotting analysis, rats were killed immediately on the second day of CPA conditioning.

\section{Drugs and antibodies}

Morphine hydrochloride was purchased from Qinghai Pharmaceutical General Factory. Sigma Aldrich supplied the naloxone hydrochloride. NSC23766 and Y27632 were obtained from Calbiochem. The antibodies against actin were purchased from Sigma-Aldrich and diluted 1:5000 for Western blot analysis.

\section{Subcellular fractionation}

Rats were anesthetized and killed by decapitation. Coronal brain sections ( $1 \mathrm{~mm}$ thick) were obtained using a rat brain slicer (Braintree Scientific Inc, Braintree, MA). Both sides of the hippocampus were punched from brain slices using a blunt-end, 17-gauge syringe needle (1-mm inner diameter). In all subsequent procedures, the tissues were maintained at $4^{\circ} \mathrm{C}$. A crude synaptosomal fraction was made according to the previous procedure ${ }^{[21]}$. Briefly, the tissue was homogenized with 10 strokes using a Teflon pestle in 10 volumes of 0.32 $\mathrm{mol} / \mathrm{L}$ sucrose. The homogenate was centrifuged at $1000 \times g$ for $10 \mathrm{~min}$, and the pellet was discarded. The supernatant was then centrifuged at $17000 \times \mathrm{g}$ for $30 \mathrm{~min}$. The resultant $\mathrm{P} 2$ pellet was washed in an equal volume of $0.32 \mathrm{~mol} / \mathrm{L}$ sucrose and recentrifuged at $17000 \times g$ for another $30 \mathrm{~min}$. The pellet was used as intact synaptosomes.

The amount of F-actin and G-actin was measured with an Actin Polymerization Assay Kit (Cytoskeleton, BK037). Briefly, the synaptosomes were resuspended in Lysis and F-actin Stabilization Buffer (Cytoskeleton, Part\# LAS01), with protease inhibitor Cocktail (1:100, Cytoskeleton, Cat \# PIC02) and ATP (Cytoskeleton, Cat \#BSA04-001), and then centrifuged at $37^{\circ} \mathrm{C}$ at $100000 \times g$ for $1 \mathrm{~h}$. The G-actin fraction (the supernatant) and the F-actin fraction (the pellet) were collected and analyzed by Western blotting. 


\section{Intracerebral microinjection}

Rats were anesthetized using sodium pentobarbital (50 $\mathrm{mg} / \mathrm{kg}$, ip), treated with atropine sulfate $(0.2 \mathrm{mg} / \mathrm{kg}$, ip), and then placed in a stereotaxic apparatus (Narishige, Tokyo, Japan) with the incisor bar set at $3.3 \mathrm{~mm}$. Rats were implanted bilaterally with guide cannulae (26 gauge) in the dorsal hippocampus (AP, $-3.5 \mathrm{~mm}$; ML, $\pm 2.5 \mathrm{~mm}$; DV, $-1.5 \mathrm{~mm}$ ). Three surgical screws were implanted into the skull as anchors. The cannulae and the screws were affixed to the skull with dental cement. Insect pins $(0.5 \mathrm{~mm}$ beyond the tip of the guide cannula) were inserted into the cannulae to maintain patency and were removed only for the infusions. All rats were given 7-10 d for postsurgical recovery before commencing behavioral training.

Bilateral microinfusions were made through 31-gauge injection cannulae ( $2.0 \mathrm{~mm}$ beyond the tip of the guide cannulae), which were connected to $10 \mu \mathrm{L}$ microsyringes mounted to the microinfusion pump (Harvard Apparatus, South Natick, MA) for no less than 2 min and given an additional 2 min for drug diffusion. Y27632 and NSC23766 were dissolved in PBS before use and were bilaterally microinjected into the dorsal hippocampus 30 and 15 min before pairing, respectively. The doses of Y27632 and NSC23766 were chosen based on pilot experiments and previous studies ${ }^{[21,22]}$.

\section{Histology}

After behavior testing, rats were deeply anesthetized with sodium pentobarbital and perfused transcardially with $0.9 \%$ saline followed by $4 \%$ paraformaldehyde in phosphatebuffered saline. The brains were removed and stored in a $30 \%$ sucrose/PBS solution for 2-3 d. Coronal sections (30 $\mu \mathrm{m}$ thick) were cut on a cryostat (Leica), stained with cresyl violet, and then examined by light microscopy to determine injection sites.

\section{Data analysis}

The data were analyzed with a two-tailed Student's $t$ tests or a one-way ANOVA followed by Newman-Keuls post hoc tests when appropriate. Differences with $P<0.05$ were considered statistically significant. The results are presented as the mean \pm SEM.

\section{Results}

CPA was accompanied by actin rearrangements in the hippocampus

CPA is a reflection of the aversion memories of morphine withdrawal with a particular environment. Our previous study revealed that the actin rearrangements in the dorsal hippocampus were involved in the formation of $\mathrm{CPA}^{[8]}$. In the present study, using a similar training paradigm, we measured the synaptic actin rearrangement dynamics in the dorsal hippocampus, that is, we tested the levels of the monomeric actin (G-actin) and the polymerized actin (F-actin) at 0.5, 1 and $2 \mathrm{~h}$ after conditioned naloxone-precipitated morphine withdrawal and compared them with the saline-pairing control group. In accordance with previous findings, a significant elevation of the ratio of F-actin to G-actin was observed in the dorsal hippocampus at $1 \mathrm{~h}$ but not at $0.5 \mathrm{~h}$ or $2 \mathrm{~h}$ (con: $100 \% \pm 9.02 \%$, $n=5 ; 0.5$ h: $99.9 \% \pm 9.2 \%, n=5 ; 1$ h: $226.4 \% \pm 37.8 \%, n=5 ; 2$ h: $134 . \% \pm 19.3 \%, F_{(3,17)}=7.292, P=0.0035$; Figure $\left.1 \mathrm{~A}\right)$ after conditioned naloxone-precipitated morphine withdrawal. However, the levels of total actin were not altered in the dorsal hippocampus at any of the times tested $\left(F_{(3,17)}=0.3149, P=0.8144\right.$; Figure $1 \mathrm{~B})$. These results demonstrated that actin rearrangements occurred in the dorsal hippocampus in response to conditioned morphine withdrawal.
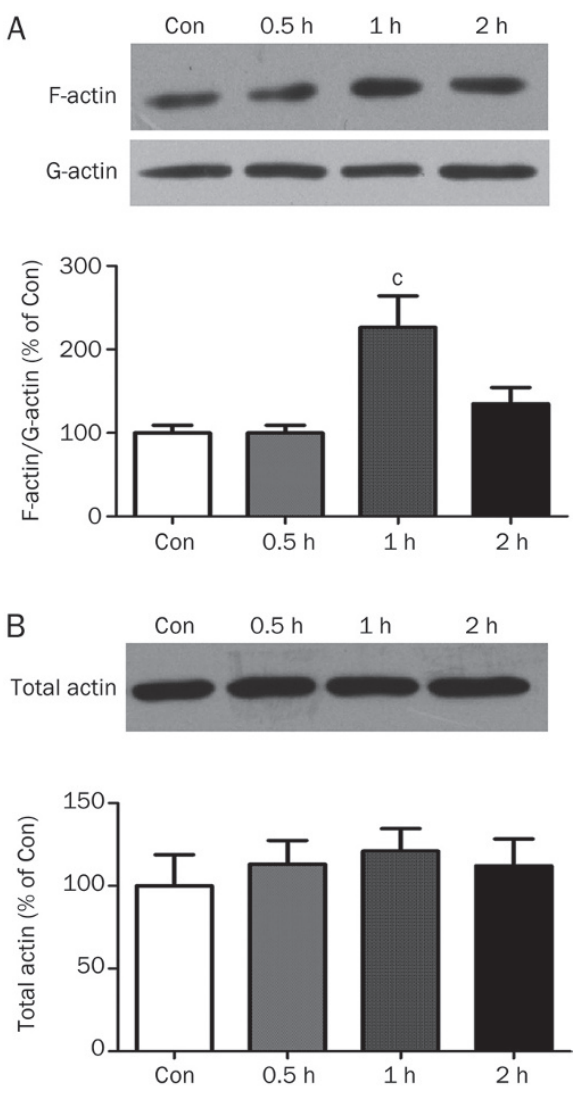

Figure 1. Effects of conditioned naloxone-precipitated morphine withdrawal on actin rearrangements in the dorsal hippocampus. (A) Conditioned morphine withdrawal induced an increase in the ratio of F-actin to G-actin in the dorsal hippocampus at $1 \mathrm{~h}$ but not at $0.5 \mathrm{~h}$ or $2 \mathrm{~h}$ after naloxone injection. (B) Total actin protein levels are similar in all groups. Values are expressed as the mean \pm SEM. ${ }^{c} P<0.01$, compared with the corresponding saline-treated control group using a one-way ANOVA with a Newman-Keuls post hoc test. Con, control.

Effects of intra-dorsal hippocampus injection of NSC23766 or Y27632 on the acquisition of conditioned place aversion induced by naloxone-precipitated morphine withdrawal

The data presented above confirm that actin polymerization in the dorsal hippocampus is involved in the aversive memory formation of conditioned morphine withdrawal. However, the molecular mechanism by which actin polymerization modu- 
lates CPA formation is still elusive. Previous studies have demonstrated that actin polymerization in the hippocampus induced by high-frequency stimulation (HFS) depends on NMDA receptor activation and can be blocked by Rho kinase inhibitors $^{[23,24]}$. Members of the Rho GTPase family, including Rho, Rac, and Cdc42, are regarded as key regulators of the actin cytoskeleton. In this study, we tried to determine which molecular event was involved in actin polymerization.

First, we determined whether the Rho GTPase family is involved in the acquisition of morphine withdrawalinduced CPA. We chose two of the most widely reported Rho GTPases, RhoA and Rac1, as our research candidates. The RhoA-ROCK inhibitor Y27632 (8.56 $\mu \mathrm{g} / 1 \mu \mathrm{L}$ per side) or the Rac1 inhibitor NSC23766 (25 $\mu \mathrm{g} / 1 \mu \mathrm{L}$ per side) was bilaterally microinfused into the dorsal hippocampus $30 \mathrm{~min}$ or $15 \mathrm{~min}$, respectively, before naloxone injection during the conditioning session. In the rats microinjected with vehicle or NSC23766 into the bilateral dorsal hippocampus, the time spent in the drug-paired compartment during the test session was $356.9 \pm 83.7 \mathrm{~s}$ and $277.4 \pm 79.3 \mathrm{~s}$, respectively, both of which were significantly shorter than the time spent during the preconditioning session $(657.2 \pm 35.6 \mathrm{~s}$ and $588.1 \pm 31.1 \mathrm{~s}$, respectively; $P<0.001$, Student's $t$ test; Figure 2A), demonstrating that CPA
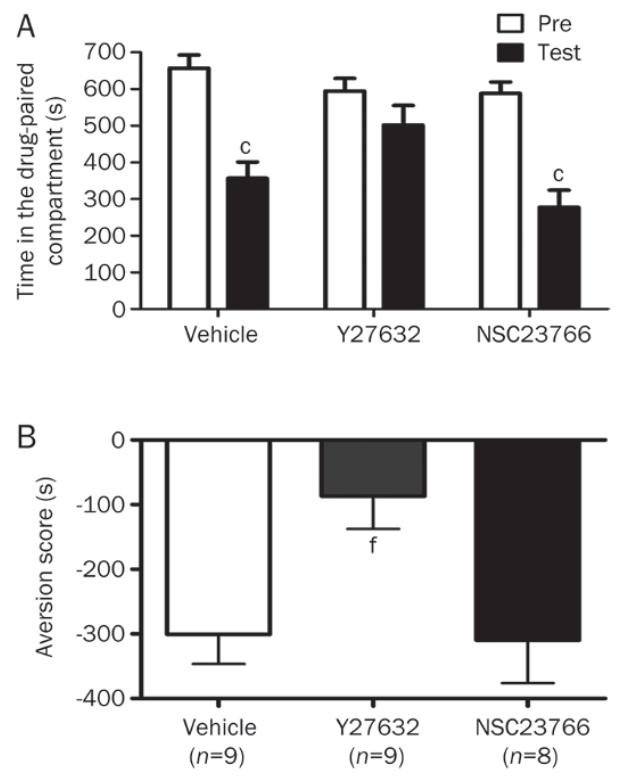

Figure 2. Effects of an intra-dorsal hippocampus infusion of Y27632 or NSC23766 on the formation of conditioned place aversion induced by naloxone-precipitated morphine withdrawal in morphine-treated rats. Y27632 (8.56 $\mu \mathrm{g} / 1 \mu \mathrm{L}$ per side), NSC23766 (25 $\mu \mathrm{g} / 1 \mu \mathrm{L}$ per side) or vehicle ( $1 \mu \mathrm{L} / \mathrm{side})$ was bilaterally microinfused into the dorsal hippocampus before pairing on the second day of the conditioning session. The columns show the time spent in the drug-paired compartment during the preconditioning (Pre) or testing (test) sessions (A) and the CPA score (B). The CPA score is defined as time in the drug-paired compartment during the testing session minus the time spent during the preconditioning session. Data are expressed as means \pm SEM. ${ }^{c} P<0.01$ compared with the corresponding preconditioning session (Student's $t$ test). ${ }^{f} P<0.01$ compared with vehicle-injected rats using a one-way ANOVA with a Newman-Keuls post hoc test. was dramatically induced by naloxone injection following a single exposure to morphine, consistent with previous studies. However, when Y27632 was microinjected into the dorsal hippocampus, there was no significant difference between the time spent in the drug-paired compartment during the testing session $(502.2 \pm 108.5 \mathrm{~s})$ and that spent during the preconditioning session $(594.5 \pm 34.5 \mathrm{~s} ; P>0.05$, Student's $t$ test; Figure 2A). CPA scores revealed an inhibition of morphine withdrawal-induced CPA behavior by the intra-dorsal hippocampus injection of Y27632 but not NSC23766 (Figure 2B). A one-way ANOVA indicated a significant difference among the groups $\left(F_{(2,25)}=5.495 ; P=0.0112\right)$. Post hoc comparison by

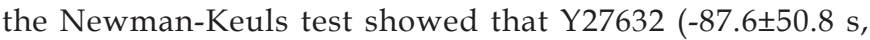
$P=0.0066)$ produced a significant attenuation of the CPA score compared with the vehicle-injected morphine-treated group $(-300.3 \pm 46.0 \mathrm{~s})$. The aversion score of the NSC23766-injected group, however, was not significantly difference compared with the vehicle-injected group $(-309.6 \pm 66.6 \mathrm{~s}, P=0.9085)$. These results demonstrated that the activity of RhoA, but not Rac1, in the dorsal hippocampus was involved in the formation of CPA.

Actin polymerization occurred in the dorsal hippocampus through the RhoA but not the Rac1 signaling pathway after conditioned morphine withdrawal

Our study has revealed that conditioned morphine withdrawal induced synaptic actin polymerization in the dorsal hippocampus of rats, as characterized by a significant elevation of polymerized F-actin and a decrease of monomeric G-actin. A previous study also showed that microinjection of the actin polymerization inhibitor, latrunculin A, into the dorsal hippocampus attenuated the CPA score ${ }^{[8]}$, demonstrating that the dorsal hippocampal actin rearrangements were involved in the formation of CPA. Because the results above showed that microinjection of the RhoA inhibitor Y27632, but not the Rac1 inhibitor NSC23766, prior to conditioned pairing in the dorsal hippocampus resulted in an attenuated CPA score, we further investigated whether RhoA, but not Rac1, was also involved in actin polymerization in the dorsal hippocampus.

The RhoA-ROCK inhibitor Y27632 (8.56 $\mu \mathrm{g} / 1 \mu \mathrm{L}$ per side) or the Rac1 inhibitor NSC23766 (25 $\mu \mathrm{g} / 1 \mu \mathrm{L}$ per side) was bilaterally microinfused into the dorsal hippocampus $30 \mathrm{~min}$ or $15 \mathrm{~min}$ before conditioned pairing, and the F-actin and G-actin levels at $1 \mathrm{~h}$ after naloxone injection were analyzed. The results are shown in Figure 3. There was a significant increase in F-actin and a decrease in G-actin detected in the vehicle-injected group $(199.5 \% \pm 29.2 \%, n=5)$ compared with the control group $(100.0 \% \pm 5.5 \%, n=5)$. Bilateral microinfusion of the Rac1 inhibitor NSC23766 (25 $\mu \mathrm{g} / 1 \mu \mathrm{L}$ per side) into the dorsal hippocampus $15 \mathrm{~min}$ before naloxone injection also resulted in a significant increase in the ratio of F-actin/G-actin $(216.9 \% \pm 24.3 \%$ of control, $n=5)$, which demonstrates that injection of NSC23766 has no effect on actin rearrangements in the dorsal hippocampus. However, bilateral microinjection of the RhoA inhibitor Y27632 (8.56 $\mu \mathrm{g} / 1 \mu \mathrm{L}$ per side) into the dorsal hippocampus $30 \mathrm{~min}$ before naloxone injection significantly 
A
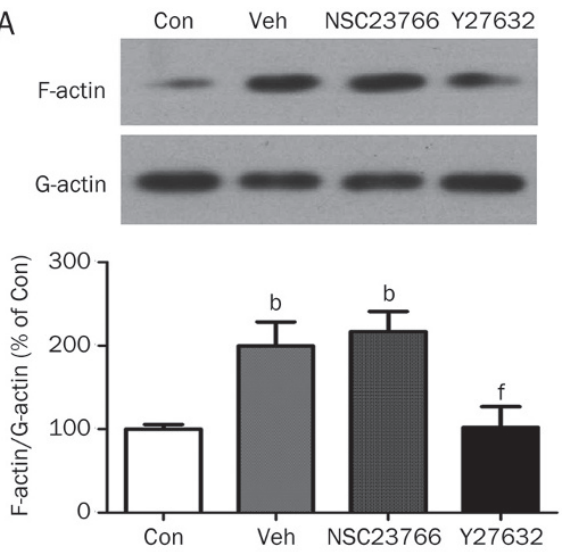

B
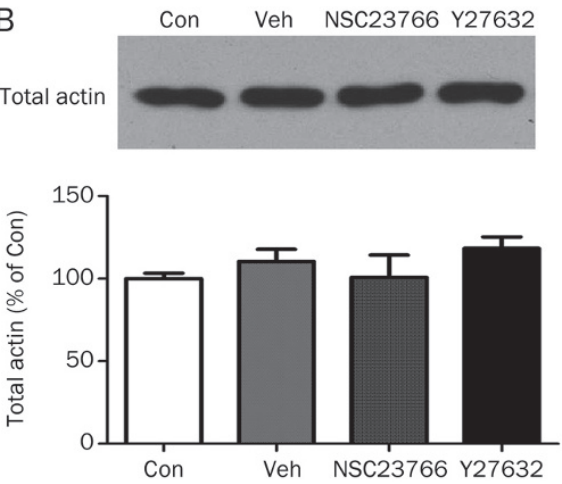

Figure 3. Effects of pre-conditioning using intra-dorsal hippocampus injections of NSC23766 (25 $\mu \mathrm{g} / 1 \mu \mathrm{L}$ per side) or Y27632 $(8.56 \mu \mathrm{g} / 1 \mu \mathrm{L}$ per side) on the ratio of F-actin to G-actin or total actin in the dorsal hippocampus at $1 \mathrm{~h}$ after CMW. (A) NSC23766 had no effect on the enhanced ratio of F-actin to G-actin, while Y27632 significantly reduced the ratio of F-actin to G-actin in the dorsal hippocampus at $1 \mathrm{~h}$ after conditioned morphine withdrawal. (B) Total actin protein levels are similar in all groups. Mean \pm SEM. ${ }^{b} P<0.05$, compared with the corresponding saline-treated control group. ${ }^{f} P<0.01$, compared with the vehicle-injected group using a one-way ANOVA with a Newman-Keuls post hoc test. Con, control.

reduced the ratio of F-actin/G-actin compared with the vehicle-injected group $\left(102 \% \pm 25.1 \%\right.$ of control, $n=5 ; F_{(3,19)}=7.368$, $P=0.0026$; Figure $3 \mathrm{~A}$ ), indicating that the RhoA pathway was involved in the actin rearrangements in the dorsal hippocampus. No significant differences in the levels of total actin were found between any of the experimental groups in the dorsal hippocampus $\left(F_{(3,19)}=1.023, P=0.0981, n=5\right.$; Figure 3B). These results indicate that the small GTPase RhoA, but not Rac1, was essential for synaptic actin polymerization in the dorsal hippocampus, consistent with our behavioral results that RhoA, but not Rac1, was involved in aversive memory formation.

\section{Histology}

The injection sites for all of the rats included in the analysis of this study are illustrated in Figure 4. The animals were killed after behavior testing, and their brains were assessed by nissl staining (Figure 4A). There was no significant sign of damage
A
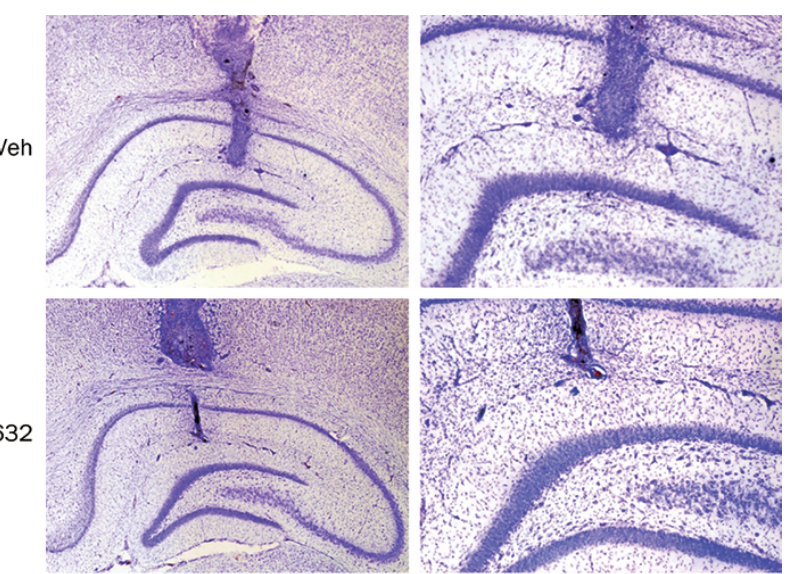

NSC23766
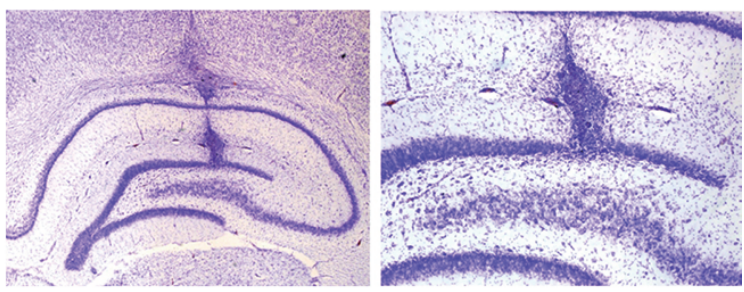

B

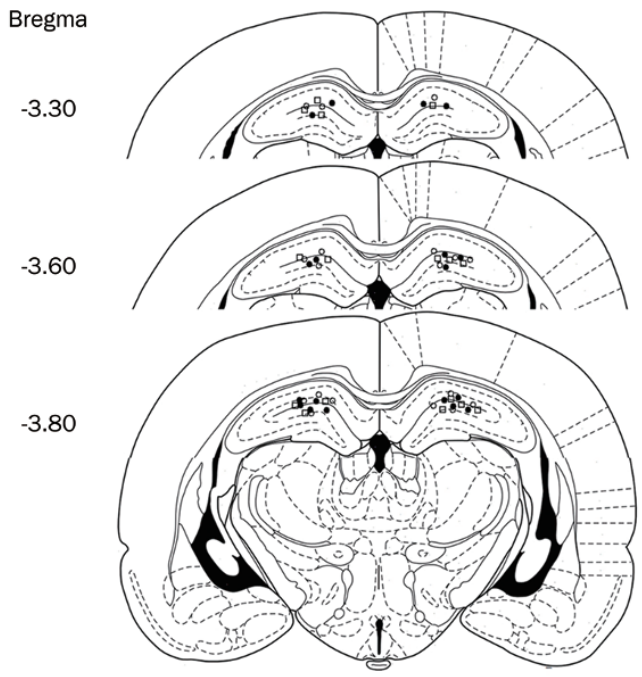

Figure 4. Nissl staining (A) and schematic representation of injection sites $(B)$ in the dorsal hippocampus for all rats included in the study (O, preconditioning microinjection of vehicle in morphine-treated rats; $\bullet$, preconditioning microinjection of Y27632 in morphine-treated rats; $\square$, preconditioning microinjection of NSC23766 in morphine-treated rats). Numbers to the left of the coronal sections represent the distance $(\mathrm{mm})$ from the bregma along the anterior-posterior axis.

found in the dorsal hippocampus in any of the three groups. All injection sites were located in the dorsal hippocampus (Figure 4B). Injection sites for the vehicle, NSC23766 and Y27632 were basically the same; thus, no difference among the three conditions resulted from differential injection sites.

\section{Discussion}

The behavioral expression of place aversion reflects an associa- 
tion between the aversive memories of drug withdrawal and the context. An extensive body of work has associated learning and memory formation (including long-term potentiation, LTP and long-term depression, LTD) with changes in synaptic structure, namely structural synaptic plasticity ${ }^{[25-27]}$. Cytoskeletal actin is the major structural component of the dendritic spine, which is a critical site for synaptic plasticity ${ }^{[28-30]}$. Actin can be rapidly polymerized to induce cytoskeletal rearrangements, and developmental studies have shown that changes in spine morphological and structural stability and motility depended on actin polymerization. Thus, actin rearrangements in dendritic spines contribute to structural and functional changes in synapses ${ }^{[23,31,32]}$ and behavioral adaptations ${ }^{[33-36]}$, especially in certain key brain structures, including the hippocampus. In support of those findings, the present study confirmed that conditioned withdrawal from acutely morphine-dependent rats resulted in actin polymerization at $1 \mathrm{~h}$ but not $0.5 \mathrm{~h}$ or $2 \mathrm{~h}$ in the dorsal hippocampus, which was characterized by a net increase in the F-actin content and a decrease in the G-actin content in the synapses. A previous study demonstrated that inhibition of actin rearrangements in the dorsal hippocampus by intra-hippocampus injections of latrunculin $\mathrm{A}$, an inhibitor of actin polymerization, after conditioned pairing significantly attenuated the CPA score ${ }^{[8]}$. These data support the notion that actin rearrangements in the dorsal hippocampus in response to conditioned morphine withdrawal are involved in aversive memory formation.

Next, we tried to understand the molecular mechanisms underlying actin polymerization in conditioned morphine withdrawal. Previous research has shown that signaling pathways involving the Rho family of small GTPases are key regulators of actin polymerization and myosin function of different aspects of neuronal morphologies ${ }^{[37,38]}$. RhoA, Rac and Cdc42 are the three best-studied Rho GTPases, which control the formation of distinct F-actin-based structures ${ }^{[39,40]}$. Studies have shown that RhoA and Rac1 are important for structural aspects of the mature neuron ${ }^{[41]}$ and are related to learning and memory ${ }^{[42,43]}$. The present study set about to explore the relationship between the small Rho GTPases and drug withdrawal-induced hippocampal actin polymerization. We tested whether RhoA and Rac1 were responsible for the actin rearrangements in the dorsal hippocampus in response to aversive memory formation of morphine withdrawal. We first determined whether RhoA or Rac1 was responsible for conditioned place aversion formation, and our results showed that the RhoA-ROCK inhibitor Y27632 significantly attenuated CPA when micro-injected into the dorsal hippocampus before conditioned pairing but not after conditioned pairing (data not show). Rats that were injected in the intra-dorsal hippocampus with the Rac1 inhibitor NSC23766 prior to conditioned pairing exhibited a strong CPA, demonstrating that RhoA, but not Rac1, was required in aversive memory acquisition. This result is in accordance with a previous study showing that genetic disruption or pharmacological inactivation of the
RhoA-ROCK signaling pathway in the hippocampus impaired long-term learning and memory in rats ${ }^{[44]}$.

Given the results showing the critical role for Rho GTPases in morphine withdrawal, we then tried to address the role of Rho GTPases in actin rearrangements in response to conditioned morphine withdrawal. Activation of Rho GTPases can lead to the binding of multiple effectors; therefore, these proteins play major roles in a number of neural functions, including the organization of the actin cytoskeleton. We hypothesized that the Rho GTPases controlled memory formation through the regulation of actin polymerization in the dorsal hippocampus, which has been confirmed to be required for CPA formation ${ }^{[8]}$. We micro-injected the RhoA inhibitor Y27632 or the Rac1 inhibitor NSC23766 into the dorsal hippocampus prior to conditioned training and examined the ratios of F-actin/G-actin at $1 \mathrm{~h}$ after naloxone injection. As we expected, inhibition of RhoA, but not Rac1, before conditioned pairing significantly blocked synaptic actin polymerization induced by conditioned morphine withdrawal. In support of this result, our previous research revealed that synaptic actin polymerization in the amygdala was dependent on the activation of the RhoA-ROCK signaling pathway, which was shown to play a critical role in CPA formation ${ }^{[21]}$. Moreover, previous work also demonstrated that actin polymerization induced by HFS in the hippocampus can be blocked by Rho kinase (ROCK) inhibitors ${ }^{[24]}$. Taken together, activation of the small Rho GTPase RhoA is most likely essential for the structural plasticity associated with experience and memory formation.

The hippocampus is a medial temporal lobe structure that has been widely reported to be critically involved in several aspects of long-term memory ${ }^{[45,46]}$. However, very few studies have addressed its role in drug withdrawal-associated aversive learning and memory. Our previous study has revealed that excitotoxic lesions of the dorsal hippocampus impaired CPA induced by morphine withdrawal ${ }^{[8,47]}$, certifying the essential role of the hippocampus in aversive memory formation. The present study further discusses the mechanisms and the involvement of dorsal hippocampal actin polymerization in CPA formation and indicates that the actin rearrangements rely on the activation of Rho GTPases. Taken together, we demonstrate the essential molecular events underlying actin rearrangements in the synapses of the dorsal hippocampus, which are responsible for the formation of aversive memories associated with drug withdrawal. Therefore, our work provides a possible pathway in the hippocampus for understanding the mechanisms of aversive memory formation.

\section{Acknowledgements}

This research was supported by the National Basic Research Program grant from the Ministry of Science and Technology of China 2009CB522005 (Jing-gen LIU), as well as grants from the Foundation of National Natural Science of China 81130087 (Jing-gen LIU), 81001424 (Yuan-yuan HOU) and the Foundation of Natural Science of Jiangshu Province SBK201241521 (Yu-hua WANG). 


\section{Author contribution}

Jing-gen LIU, Yao LIU, and Tao XI designed the research work; Yao LIU, Jun WANG, Yu-Hua WANG, and Yuan-yuan HOU performed the experiments; Yao LIU analyzed the data and wrote the manuscript; Jing-gen LIU revised the manuscript.

\section{References}

1 Kenny PJ. Brain reward systems and compulsive drug use. Trends Pharmacol Sci 2007; 28: 135-41.

2 Robinson TE, Berridge KC. The neural basis of drug craving: An incentive-sensitization theory of addiction. Brain Res Rev 1993; 18: 247-91

3 Hutcheson DM, Everitt BJ, Robbins TW, Dickinson A. The role of withdrawal in heroin addiction: enhances reward or promotes avoidance? Nat Neurosci 2001; 4: 943-7.

4 Mucha RF, van der Kooy D, O'Shaughnessy M, Bucenieks P. Drug reinforcement studied by the use of place conditioning in rat. Brain Res 1982; 243: 91-105.

5 Squire, Larry R. Memory and the hippocampus: A synthesis from findings with rats, monkeys, and humans. Psychol Rev 1992; 99: 195-231.

6 Jarrard LE. On the role of the hippocampus in learning and memory in the rat. Behav Neural Biol 1993; 60: 9-26.

7 O'Reilly RC, Rudy JW. Conjunctive representations in learning and memory: Principles of cortical and hippocampal function. Psychol Rev 2001; 108: 311-45.

8 Hou YY, Lu B, Li M, Liu Y, Chen J, Chi ZQ, et al. Involvement of actin rearrangements within the amygdala and the dorsal hippocampus in aversive memories of drug withdrawal in acute morphine-dependent rats. J Neurosci 2009; 29: 12244-54.

9 Weeber EJ, Beffert U, Jones C, Christian JM, Förster E, Sweatt JD, et al. Reelin apoE receptors cooperate to enhance hppocampal synaptic plasticity and learning. J Biol Chem 2002; 277: 39944-52.

10 Engert F, Bonhoeffer T. Dendritic spine changes associated with hippocampal long-term synaptic plasticity. Nature 1999; 399: 66-70.

11 Fischer M, Kaech S, Knutti D, Matus A. Rapid actin-based plasticity in dendritic spines. Neuron 1998; 5: 847-54.

12 Yuste R, Bonhoeffer T. Morphological changes in dendritic spines associated with long-term synaptic plasticity. Annu Rev Neurosci 2001; 24: 1071-89.

13 Halpain S. Actin and the agile spine: how and why do dendritic spines dance? Trends Neurosci 2000; 23: 141-6.

14 Toda S, Shen HW, Peters J, Cagle S, Kalivas PW. Cocaine increases actin cycling: effects in the reinstatement model of drug seeking. J Neurosci 2006; 26: 1579-87.

15 Rex CS, Gavin CF, Rubio MD, Kramar EA, Chen LY, Jia Y, et al. Myosin Ilb regulates actin dynamics during synaptic plasticity and memory formation. Neuron 2010; 67: 603-17.

16 Lamprecht R, LeDoux J. Structural plasticity and memory. Nat Rev Neurosci 2004; 5: 45-54.

17 Luo L. Actin cytoskeleton regulation in neuronal morphogenesis and structural plasticity. Annu Rev Cell Dev Biol 2002; 18: 601-35.

18 Hall A, Nobes CD. Rho GTPases: molecular switches that control the organization and dynamics of the actin cytoskeleton. Philosophical Transactions Royal Soc 2000; 355: 965-70.

19 Hall A. Rho GTPases and the actin cytoskeleton. Science 1998; 23: 509-14.

20 Diana G, Valentini G, Travaglione S, Falzano L, Pieri M, Zona C, et al. Enhancement of learning and memory after activation of cerebral Rho
GTPases. Proc Natl Acad Sci U S A 2007; 104: 636-41.

21 Liu Y, Zhou QX, Hou YY, Lu B, Yu C, Chen J, et al. Actin polymerizationdependent increase in synaptic Arc/Arg3.1 expression in the amygdala is crucial for the expression of aversive memory associated with drug withdrawal. J Neurosci 2012; 32: 12005-17.

22 Zhang QG, Wang R, Han D, Dong Y, Brann DW. Role of Rac1 GTPase in JNK signaling and delayed neuronal cell death following global cerebral ischemia. Brain Res 2009; 1265: 138-47.

23 Fukazawa Y, Saitoh Y, Ozawa F, Ohta Y, Mizuno K, Inokuchi K. Hippocampal LTP is accompanied by enhanced F-actin content within the dendritic spine that is essential for late LTP maintenance in vivo. Neuron 2003; 38: 447-60.

24 Huang F, Chotiner JK, Steward O. Actin polymerization and ERK phosphorylation are required for Arc/Arg3.1 mRNA targeting to activated synaptic sites on dendrites. J Neurosci 2007; 27: 9054-67.

25 Sweatt JD. Mitogen-activated protein kinases in synaptic plasticity and memory. Curr Opin Neurobiol 2004; 14: 311-7.

26 Bailey $\mathrm{CH}$, Kandel ER. Structural changes accompanying memory storage. Annu Rev Physiol 1993; 55: 397-426.

27 Geinisman Y. Structural synaptic modifications associated with hippocampal LTP and behavioral learning. Cerebral Cortex 2000; 10: 952-62.

28 Matus A, Ackermann M, Pehling G, Byers HR, Fujiwara K. High actin concentrations in brain dendritic spines and postsynaptic densities. Proc Natl Acad Sci U S A 1982; 79: 7590-4.

29 Matus A. Actin-based plasticity in dendritic spines. Science 2000; 290: 754-8.

30 Fifkova E, Morales M. Actin matrix of dendritic spines, synaptic plasticity, and long-term potentiation. Int Rev Cytol 1992; 139: 267 307.

31 Zhou Q, Homma KJ, Poo MM. Shrinkage of dendritic spines associated with long-term depression of hippocampal synapses. Neuron 2004; 44: 749-57.

32 Zito K, Knott G, Shepherd GM, Shenolikar S, Svoboda K. Induction of spine growth and synapse formation by regulation of the spine actin cytoskeleton. Neuron 2004; 44: 321-34.

33 Fischer A, Sananbenesi F, Schrick C, Spiess J, Radulovic J. Distinct roles of hippocampal de novo protein synthesis and actin rearrangement in extinction of contextual fear. J Neurosci 2004; 24: 1962-6.

34 Offenhauser N, Castelletti D, Mapelli L, Soppo BE, Regondi MC, Rossi $\mathrm{P}$, et al. Increased ethanol resistance and consumption in Eps8 knockout mice correlates with altered actin dynamics. Cell 2006; 127: 213-26.

35 Rothenfluh A, Threlkeld RJ, Bainton RJ, Tsai LT, Lasek AW, Heberlein $U$. Distinct behavioral responses to ethanol are regulated by alternate RhoGAP18B isoforms. Cell 2006; 127: 199-211.

36 Toda S, Shen HW, Peters J, Cagle S, Kalivas PW. Cocaine increases actin cycling: effects in the reinstatement model of drug seeking. J Neurosci 2006; 26: 1579-87.

37 Kaibuchi K, Kuroda S, Amano M. Regulation of the cytoskeleton and cell adhesion by the Rho family GTPases in mammalian cells. Annu Rev Biochem 1999; 68: 459-86.

38 Hotchin NA, Hall A. Regulation of the actin cytoskeleton, integrins and cell growth by the Rho family of small GTPases. Cancer Surv 1996; 27: 311-22.

39 Nobes CD, Hall A. Rho, rac, and cdc42 GTPases regulate the assembly of multimolecular focal complexes associated with actin stress fibers, lamellipodia, and filopodia. Cell 1995; 81: 53-62.

40 Moorman JP, Luu D, Wickham J, Bobak DA, Hahn CS. A balance of signaling by Rho family small GTPases RhoA, Rac1 and Cdc42 coordinates cytoskeletal morphology but not cell survival. Oncogene 
1999; 18: 47-57.

41 Dash PK, Orsi SA, Moody M, Moore AN. A role for hippocampal RhoROCK pathway in long-term spatial memory. Biochem Biophys Res Commun 2004; 322: 893-8.

42 Haditsch U, Leone DP, Farinelli M, Grashoff AC, Brakebusch C, Mansuy $\mathrm{IM}$, et al. A central role for the small GTPase Rac1 in hippocampal plasticity and spatial learning and memory. Mol Cell Neurosci 2009; 41: 409-19.

43 Oh D, Han S, Seo J, Lee JR, Choi J, Groffen J, et al. Regulation of synaptic Rac1 activity, long-term potentiation maintenance, and learning and memory by BCR and ABR Rac GTPase-activating proteins. J Neurosci 2010; 30: 14134-44.
44 Diana G, Valentini G, Travaglione S, Falzano L, Pieri M, Zona C, et al. Enhancement of learning and memory after activation of cerebral Rho GTPases. Proc Natl Acad Sci U S A 2007; 104: 636-41.

45 Izquierdo I, Medina JH. Memory formation: the sequence of biochemical events in the hippocampus and its connection to activity in other brain structures. Neurobiol Learn Mem 1997; 68: 285-316.

46 Henke K, Buck A, Weber B, Wieser HG. Human hippocampus establishes associations in memory. Hippocampus 1997; 7: 249-56.

47 Hou YY, Liu Y, Kang S, Yu C, ZQ Chi, Liu JG. Glutamate receptors in the dorsal hippocampus mediate the acquisition, but not the expression, of conditioned place aversion induced by acute morphine withdrawal in rats. Acta Pharmacol Sin 2009; 30: 1385-91. 